\title{
Superdirected beam of the backward volume spin wave
}

\author{
Alexander Yu. Annenkov, Sergey V. Gerus and Edwin H. Lock* \\ Kotel'nikov Institute of Radio Engineering and Electronics of RAS (Fryazino branch), 141190 Vvedensky sq.1, Fryazino, Moscow \\ region, Russia
}

\begin{abstract}
Visualized patterns of the backward volume spin wave (BVSW) excited by arbitrarily oriented linear transducer in tangentially magnetized ferrite film are investigated experimentally in the plane of ferrite film for the case where the transducer length $D$ is much larger than the wavelength $\lambda_{0}$. Superdirected BVSW beam having zero angular width and minimal smearing of the beam energy along the film surface is observed experimentally. Thus, it is proved that such phenomenon as "superdirected propagation of the wave" exists in the nature.
\end{abstract}

\section{Introduction}

Some years ago it was investigated theoretically a twodimensional diffraction patterns arising in the far-field region of a ferrite slab plane for the common geometry, where a plane surface spin wave with noncollinear group and phase velocities is incident on the wide slit in opaque screen with arbitrary orientation [1,2]. Using magnetostatic approximation [3], it was found, that as a distinct from isotropic media the angular width $\Delta \psi$ of the diffracted spin wave beam is defined not only by the ratio $\lambda / D$ (here $\lambda$ is the incident wavelength and $D$ is the slit length), but also by the mathematical properties of isofrequency dependence (ID) ${ }^{\mathrm{a}}$ for the diffracted wave. The general formula for the angular width $\Delta \psi$ of a diffracted beam was derived as a result of this investigation. It was shown that this formula is valid not only for various types of spin waves, but also for other waves, propagating in various anisotropic media and structures (including metamaterials). As follows from the obtained formula, the angular width of the beam in anisotropic media can not only take values greater or less than $\lambda_{0} / D$, but also can be equal to zero under certain conditions [2]. Thus, analyzing mathematical properties of the wave ID in the certain anisotropic medium or structure, one can find out the wave vector orientation for which superdirected beams (i.e. nonexpanding beams with zero angular width) can be observed experimentally.

Using the analysis of the spin wave ID it was shown in the work [4] that superdirected beams of the backward volume spin waves (BVSW) can be observed in the free ferrite slab too. Mention must be made, that the similar results for BVSW was predicted also in [5], where for superdirected propagation of the beam it was used the terms "focusing" and "caustic propagation".

a ID is also known as "section of wavevector surface", "section of the isoenergy surface" and "equifrequency line".
In order to confirm validity of theoretical results described above, a visualized patterns of the surface spin wave excited by arbitrarily oriented linear transducer were investigated and the angular width of spin wave beams was measured [6]. It was found in the [6] that experimental results are in good agreement with theoretical calculations, basing on $[1,2]$. Moreover, superdirected surface spin wave beam was observed experimentally [6].

Below we present the experimental visualized pattern of superdirected BVSW beam excited by arbitrarily oriented linear transducer.

\section{Experimental and calculated results}

The general scheme of experimental setup is shown in Fig. 1. Propagation of spin wave beams was studied in yttrium iron garnet (YIG) film 5, grown on a gadolinium gallium garnet (GGG) substrate with a diameter of 76 $\mathrm{mm}$ and a thickness of $0.5 \mathrm{~mm}$. YIG film with thickness $s=48.5 \mu \mathrm{m}$ and magnetization $4 \pi M_{0}=1800 \mathrm{Gs}$ was magnetized to saturation by the tangential uniform magnetic field $\mathbf{H}_{\mathbf{0}}$ with a value $H_{0}=500 \mathrm{Oe}$.

Microwave signal, propagating as a spin wave in the YIG film from input transducer 6 to output transducer 4 , was generated and received by the device 1 , measuring complex transmission coefficient. Then received analog signal was transformed by analog-to-digital converter and was analyzed by special computer 2. Input transducer 6 , exciting spin wave beam, and output receiving transducer 4 were made of gold-plated tungsten wire of $12 \mu \mathrm{m}$ thick. Exciting transducer 6 has the length $D=5 \mathrm{~mm}$ and receiving transducer 4 has the aperture $L \sim 0.5 \mathrm{~mm}$. Two identical systems had provided in-plane displacement of the receiving transducer in two orthogonally directions along YIG film surface. Thus, receiving transducer equipped also by the

* Corresponding author: edwin@ms.ire.rssi.ru 
special position sensors 3 was turned into movable scanning probe, directed by computer 2 .

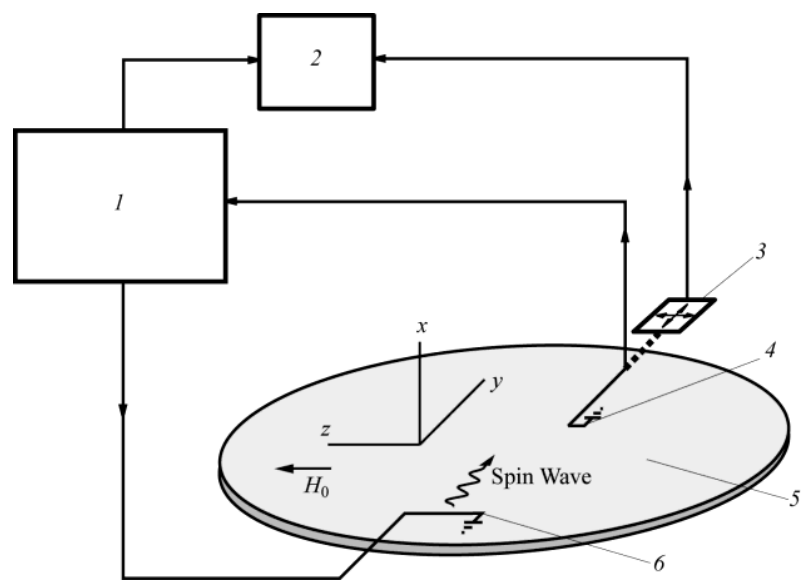

Fig. 1. Schematic view of the experimental setup: 1 - device for the measuring of complex transmission coefficient, 2 computer, 3 - sensors of the transducer position, 4 - receiving transducer (probe), 5 - YIG film, 6 -linear transducer exciting spin wave.

The distribution of spin wave beam in the plane of the yttrium iron garnet film is studied experimentally by means of the probe scanning method, that was used earlier in [7 - 9]. Recently this method was improved cardinally [10] and now it is possible to visualise distribution of amplitude and phase of spin wave along the film surface by means of computer signal processing (for more detail see [10]). Mention must be made that dipole spin wave distribution may occupy a large area compared with YIG film surface of $76 \mathrm{~mm}$ in diameter. Therefore, this method is appear much more effective for dipole spin waves than the similar well known method based on the Brillouin scattering of light by the spin wave [11], while the last method is widely used to study spin wave distribution in the ferrite film plane [12-14].

Note, that the theory [2] is also valid for the case, where spin wave beam is excited by arbitrary oriented linear transducer (this assumption is justified in section 9 of [2]). In this case general formula (30) in [2] for angular beam width $\Delta \psi$ is simplified to the next form

$$
\Delta \psi=\left(\lambda_{0} / D\right)\left|d \psi / d \varphi\left(\varphi_{0}\right)\right|
$$

Here angle $\varphi_{0}$ is orientation of the wave vector $\mathbf{k}_{\mathbf{0}}$ respect to the $y$ axis (or angle $\varphi_{0}$ may be considered as orientation of transducer's aperture line respect to the vector $\mathbf{H}_{\mathbf{0}}$ ); angles $\varphi$ and $\psi$ are orientations for an arbitrary wave vector $\mathbf{k}$ and a corresponding group velocity vector $\mathbf{V}$ respectively; $d \psi / d \varphi\left(\varphi_{0}\right)$ - the derivative value, corresponding to the point of ID in which the wave vector $\mathbf{k}_{\mathbf{0}}$ is ended (for more detail see section 8 in [2]).

Characterizing spin wave beams it is convenient to determine both their absolute angular width $\Delta \psi$ and their relative angular width $\sigma$, which is equal to the ratio between the angular width $\Delta \psi$ (in radians) and $\Delta \psi_{\text {isotr }}=$ $\lambda_{0} / D$ (the angular width of the similar diffracted beam in isotropic medium, in radians):

$$
\sigma=\Delta \psi / \Delta \psi_{\text {isotr }}=\Delta \psi /\left(\lambda_{0} / D\right)=d \psi / d \varphi\left(\varphi_{0}\right)
$$

From the physical standpoint, relative angular width $\sigma$ shows how much $\Delta \psi$ is smaller (or greater) than the absolute angular width $\Delta \psi_{\text {isotr }}$ for the similar beam in isotropic media.

Formula (1) shows, that the angular beam width $\Delta \psi$ is determined not only by the ratio $\lambda_{0} / D$, but also by the derivative value $d \psi / d \varphi\left(\varphi_{0}\right)$ characterizing the curvature of ID. So if one can find the structure (medium) and geometry (excitation topology), at which $d \psi / d \varphi\left(\varphi_{0}\right)=0$, then one can observe superdirected (nonexpanding) beam with angular width $\Delta \psi=0$, i.e. the beam retaining constant absolute width during its propagation!

As it is found in [4], the values $d \psi / d \varphi\left(\varphi_{0}\right)$ and $\sigma$ can be equal to zero for certain wave vector orientations $\varphi_{0}$, corresponding to the inflection points of BVSW ID (Fig.2). In these points vectors $\mathbf{k}$ and $\mathbf{V}$ are noncollinear. For example, if $f_{0}=2800 \mathrm{MHz}$ (curve 4 in Fig.2), then $\sigma=0$ at $\varphi_{0}= \pm 17^{\circ}$ and at $\varphi_{0}=180^{\circ} \pm 17^{\circ}$ (for more detail see also fig. 2 - 4 in [4]).

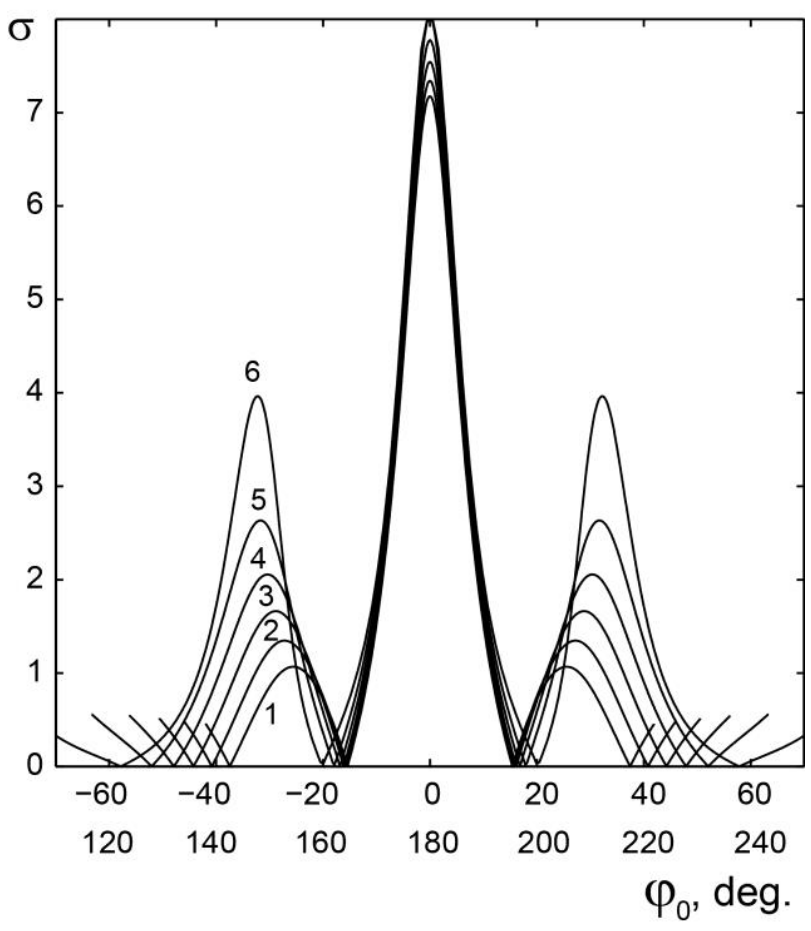

Fig. 2. Relative angular width $\sigma$ of BVSW beams versus angle $\varphi_{0}$ for various frequencies: $1-2500,2-2600,3-2700,4-$ 2800, 5 - 2900, 6 - $3000 \mathrm{MHz}$.

So to observe superdirected BVSW beam corresponding to the case $\sigma=d \psi / d \varphi\left(\varphi_{0}\right)=0$, we had excited BVSW with frequency $f_{0}=2800 \mathrm{MHz}$ and transducer orientation $\varphi_{0}=180^{\circ}-17^{\circ}=163^{\circ}$ (at which the ID of BVSW had the inflection point with $d \psi / d \varphi\left(\varphi_{0}\right)$ $=0$ ). At this ID point the wave had wave number $k_{0}=$ $118 \mathrm{~cm}^{-1}$, wave length $\lambda_{0}=532 \mu \mathrm{m}$ and ratio $\lambda_{0} / D=$ 0.106 . Visualized pattern corresponding to the amplitude distribution of the beam for this BVSW in the YIG film plane is shown in Fig. 3.

The change of color in Fig.3 corresponds to the change of spin wave amplitude about $3 \mathrm{~dB}$. It should be 
noted, that in isotropic media such beam (with ratio $\lambda_{0} / D$ $=0.106)$ would have angular width about $6.1^{\circ}$, whereas in Fig.3 spin wave beam has absolute angular width $\Delta \psi_{\text {exp }}=0.5^{\circ}$ and relative angular width $\sigma_{\exp }=0.08$ ! These experimentally measured values $\Delta \psi_{\text {exp }}$ and $\sigma_{\text {exp }}$ are slightly differ from calculated zero values, because of as a distinct from the light the spin wave can propagate only at a maximal distance ${ }^{\mathrm{b}} \sim 50 \mathrm{~mm}$ and so it is possible to measure absolute angular width with accuracy about $0.3^{\circ}$ by means of a probe with aperture of $0.5 \mathrm{~mm}$ (minimal angle $\Delta \psi_{\text {exp }}=0.5 / 50 / 2=0.005$ radian $\left.\approx 0.3^{\circ}\right)$. Since the superdirected wave beam retains its absolute width during propagation, the direction $\psi_{0}$ of the energy transfer for this beam is measured with the greatest possible accuracy $\psi_{0}=59.2^{\circ} \pm 0.3^{\circ}$. Mention must be made that the using of the probe with a smaller aperture does not increase the accuracy of measurement because at the large distances the probe sensitivity is decreased proportionally to the aperture size.

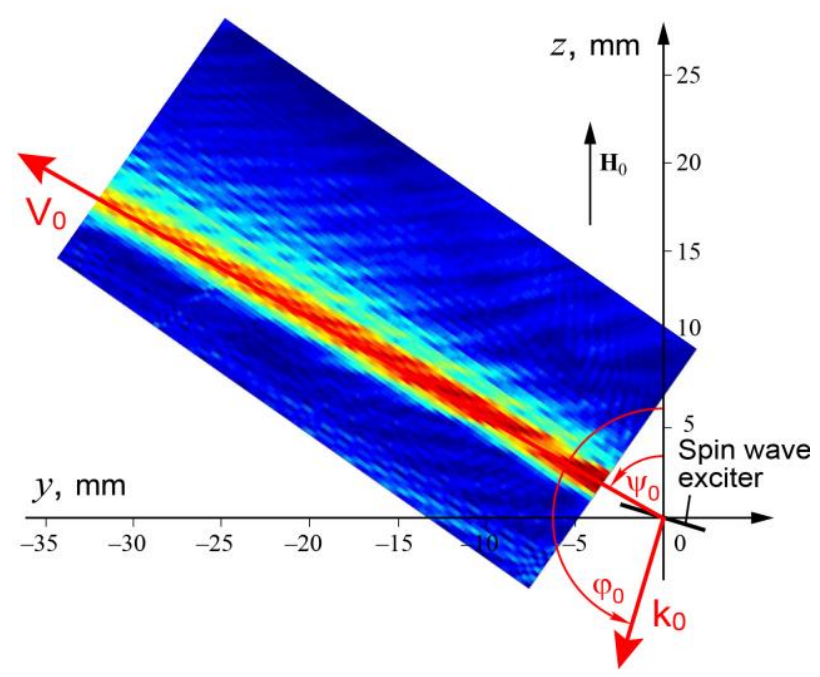

Fig. 3. Amplitude distribution of superdirected beam of BVSW in the YIG film plane (experiment) for frequency $f_{0}=2800$ $\mathrm{MHz}$, transducer orientation $\varphi_{0}=163^{\circ}, k_{0}=118 \mathrm{~cm}^{-1}$ (wave length $\lambda_{0}=532 \mu \mathrm{m}$ ), ratio $\lambda_{0} / D=0.106$ and direction of the energy transfer $\psi_{0}=59.2^{\circ}$.

\section{Summary}

Patterns of BVSW beams excited by arbitrarily oriented linear transducer in tangentially magnetized ferrite film are studied experimentally in the plane of ferrite film for the case, where the transducer length $D$ is much larger than the wavelength $\lambda_{0}$. In the study there is used the probe scanning method, that give possibility to visualise the amplitude distribution and phase distribution of the spin wave along the film surface. The angular beam width of spin waves is measured experimentally and calculated theoretically by means of the general formula for the angular beam width in anisotropic media [2].

\footnotetext{
${ }^{\mathrm{b}}$ The maximal distance value $(\sim 50 \mathrm{~mm})$ is defined by the spin wave dissipation, that is much larger than for the light wave in the lot of transparent medium.
}

It was proved experimentally that as a distinct from the beams in isotropic media the angular beam width $\Delta \psi$ of BVSW is not constant value: it depends on transducer orientation $\varphi_{0}$ and can take values greater or smaller than the ratio $\lambda_{0} / D$ (where $\lambda_{0}$ is exited wavelength and $D$ is the exciter length). Moreover, it is found such transducer orientation, at which the angular beam width $\Delta \psi$ is about zero (it means physically, that the beam retains its absolute width during propagation). Thus it was shown that such phenomenon as "superdirected propagation of the waves" exists in the nature. This phenomenon takes place when the orientation $\varphi_{0}$ of excited wave vector $\mathbf{k}_{\mathbf{0}}$ (or the transducer orientation) corresponds to the ID inflexion point. The direction $\psi_{0}$ of the energy transfer for this beam can be measured with the greatest possible accuracy. The comparison between calculated and measured values of angular beam widths both for BVSW and for surface spin waves (see [6]) demonstrates good agreement and gives us hope that the same diffractive phenomenon may take place in the other anisotropic media and structures.

The work is supported by Russian Foundation for Basic Research (project No. 17-07-00016).

\section{References}

1. E.H. Lock, Cornell University Library: https://arxiv.org/abs/1112.3929 (2011)

2. E.H. Lock, Physics-Uspekhi, 55, 1239 (2012).

3. R.W. Damon, J.R. Eshbach. J. Phys. Chem. Solids, 19, 308 (1961).

4. E.G. Lokk. J. of Communications Techn. Electron. 60, 97 (2015).

5. V. Veerakumar, R.E. Camley, Phys. Rev. B. 74, 214401 (2006).

6. A.Yu. Annenkov, S.V. Gerus, E.H. Lock, Cornell University Library: https://arxiv.org/abs/1611.09595 (2016)

7. V.I. Zubkov, E.G. Lokk, V.I. Shcheglov. Radiotekhnika i elektronika. 35, 1617 (1990).

8. A.V. Vashkovsky, V.I. Zubkov, E.H. Lock, V.I. Shcheglov. IEEE Trans. on Magn. 26, 1480 (1990).

9. A.Yu. Annenkov, I.V. Vasil'ev, S.V. Gerus, S.I. Kovalev. Tech. Phys. 40, 330 (1995).

10. A.Yu. Annenkov, S.V. Gerus. J. of Communications Techn. Electron. 57, 519 (2012).

11. S.O. Demokritov and V.E. Demidov. IEEE Trans. on Magn. 44, 6 (2008).

12. A. V. Sadovnikov et al. Appl. Phys. Lett. 107, 202405 (2015).

13. A. V. Sadovnikov et al. Appl. Phys. Lett. 108, 172411 (2016).

14. A. V. Sadovnikov et al. Phys. Rev. B. 96, 144428 (2017). 